\title{
Using Electronic Health Record-Based Tools To Screen for Bipolar Disorder in Primary Care Patients With Depression
}

\author{
James M. Gill, MD, MPH, Ying Xia Chen, MS, Angela Grimes, MS, \\ and Michael S. Klinkman, MD, MS
}

Purpose: National guidelines recommend screening all persons with depression for bipolar disorder (BPD); one way to facilitate screening is through the use of electronic health records (EHRs). This study examined the impact of an EHR-based screening and decision support tool on diagnosis and treatment of BPD among patients diagnosed with depression in primary care offices.

Methods: This nonrandomized, controlled trial was conducted in a national network of offices using EHRs. The intervention included a screening instrument and other tools for diagnosis and management of BPD, which were embedded into the EHR. This instrument automatically activated when a patient with a diagnosis of depression but no diagnosis of BPD was seen in the office. The primary outcomes were the rates of new diagnoses of BPD and prescription of new BPD medications during the 6-month study period (April to October 2009).

Results: Twenty-one offices with 75 clinicians and 8355 adult patients with depression composed the intervention group, whereas 17 offices with 81 clinicians and 8799 adult patients with depression served as the comparison group. The screening tool was used with $47.5 \%$ of intervention patients, of whom $2.5 \%$ scored at high or very high risk for BPD. Intervention patients were more likely than comparison patients to be newly diagnosed with BPD $(1.11 \%$ vs. $0.36 \% ; P<.01)$ and be prescribed new BPD medications $(1.85 \%$ vs. $1.19 \% ; P<.01)$.

Conclusions: The study suggests that EHR-based tools can be useful for screening and management of BPD for patients with depression in primary care offices. (J Am Board Fam Med 2012;25:283-290.)

Keywords: Bipolar Disorder, Depression, Electronic Health Record, Mental Health, Primary Health Care, Quality of Health Care

Bipolar disorder is increasingly being recognized as a common psychiatric condition, affecting up to $5 \%$ of the US population. ${ }^{1}$ Bipolar disorder can be difficult to distinguish from unipolar depression ${ }^{2,3}$ because many patients present with primarily depres-

This article was externally peer reviewed.

Submitted 11 July 2011; revised 19 September 2011; accepted 23 September 2011.

From the Delaware Valley Outcomes Research, Newark, DE (JMG, YXC, AG); the Department of Family and Community Medicine, Thomas Jefferson University, Philadelphia, PA (JMG); and the Department of Family Medicine and Department of Psychiatry, University of Michigan, Ann Arbor (MSK).

Funding: Funding for this study was provided by Wyeth Pharmaceuticals.

Conflict of interest: none declared.

Corresponding author: James M. Gill, MD, MPH, 17 Henderson Hill Road, Newark, DE 19711 (E-mail: gillj@ dvoresearch.com). sive symptoms. Misdiagnosis is especially problematic because treatment with antidepressant medications can sometimes precipitate manic episodes and lead to worse outcomes. ${ }^{4}$

Misdiagnosis and inappropriate treatment can be a particularly difficult problem in primary care settings, which is where most patients with depression are identified and treated. ${ }^{5,6}$ Increased efforts to enhance detection of depressive disorders in primary care have led to increased use of screening and case-finding tools in routine primary care practice, but these efforts may identify patients with bipolar disorder and depressed mood as well as depressive disorder. Recent studies by Smith et $\mathrm{al}^{7}$ and Tafalla et $\mathrm{al}^{8}$ suggest that up to $20 \%$ of primary care patients with a current diagnosis of major depressive disorder meet diagnostic criteria for bi- 
polar disorder. Because of this high potential for misdiagnosis of bipolar as unipolar depression, experts have recommended screening for bipolar disorder in all patients with a diagnosis of depression, especially in primary care settings. ${ }^{1}$ This additional screening burden would be difficult to accommodate in primary care, where time pressure is severe and competing demands are high. ${ }^{9,10}$ One way to minimize this additional practice burden would be the use of automated screening protocols embedded in electronic health records (EHRs). An EHR could provide one-click access to appropriate screening or case-finding tools, or it could provide more sophisticated screening assistance to clinicians by automatically identifying patients with a diagnosis of depression then prompting the clinician to screen for bipolar disorder. EHRs with these types of screening prompts have been shown to improve screeningincluding for cancer ${ }^{11-14}$ and hyperlipidemia ${ }^{14}$ among the general population. EHRs also have been used to facilitate screening for depression in primary care settings. ${ }^{15-17}$ However, no studies to date have examined the impact of EHR-based interventions to screen for bipolar disorder in primary care settings.

The purpose of this study was to examine the impact of a bipolar screening tool that was incorporated into the EHR for use at the point of care in primary care settings. The study examined the impact of this tool on new diagnoses of bipolar disorder and on treatment for bipolar disorder for patients with a diagnosis depression.

\section{Methods}

\section{Setting and Population}

This controlled, prospective study was conducted within a national practice-based research network (PBRN) called the Centricity Health care User Research Network (CHURN). CHURN is a network of physicians and other clinicians in ambulatory practices that use a particular outpatient HER (Centricity EMR, GE Healthcare, Waukesha, WI) and that have agreed to share data and participate in quality of care studies. CHURN members share data through a data consortium called the Medical Quality Improvement Consortium (MQIC). Each office that participates in MQIC regularly uploads de-identified clinical data into a central secure repository. These data include demographic information, medications and prescriptions, diagnoses or problems, laboratory results, and other clinical data such as blood pressure, weight, and physical examination findings. The data are then cleaned, standardized, and put into a central data repository. This data repository is used by CHURN for retrospective studies of quality of care $\mathrm{e}^{18-22}$ as well as interventional studies to improve quality of care. ${ }^{23,24}$

Offices that participate in both CHURN and MQIC were invited to participate in this study if they were primary care offices that cared for adults (family medicine, general internal medicine, or general practice) and if they had been using the Centricity EMR for at least 1 year. Offices were given the option of participating either in the intervention group or in the comparison group. The intervention group consisted of 21 offices from 10 states, ranging in size from 1 to 8 clinicians (mean, 4 clinicians; median, 3 clinicians). The comparison group consisted of 17 offices from 5 states, with a range of 1 to 13 clinicians (mean, 5 clinicians; median, 4 clinicians). The clinicians included all physicians and mid-level clinicians (nurse practitioners or physician assistants) practicing primary care at least 8 hours per week. There were a total of 75 intervention clinicians and 81 comparison clinicians. Comparison clinicians were aware that they were participating in a study related to bipolar disorder but were not told about the details of the study.

The patients included in the study were all active patients of the participating clinicians who had a diagnosis of unipolar depression but no diagnosis of bipolar disorder at the start of the study period. Active patients were defined as those with at one least office visit during the year before the study and at least one office visit during the 6-month study period. Depression was defined by the EHR problem list, including major depressive disorder (International Classification of Diseases 9 [ICD9] codes 296.2, 296.3), depressive psychosis (298.0), depression not otherwise specified (311), dysthymic disorder (300.4), depression with anxiety (300.4), or prolonged situational depression (309.1) Bipolar disorder was also defined by the EHR problem list (ICD9 codes 296.0 to 296.1 and 296.4 to $296.8 \mathrm{x}$ ).

\section{Intervention}

The intervention was a clinical decision support (CDS) tool that was embedded in the EHR (see Figure 1). The main component of the tool was a screening instrument for bipolar disorder con- 
Figure 1. Bipolar screening form.

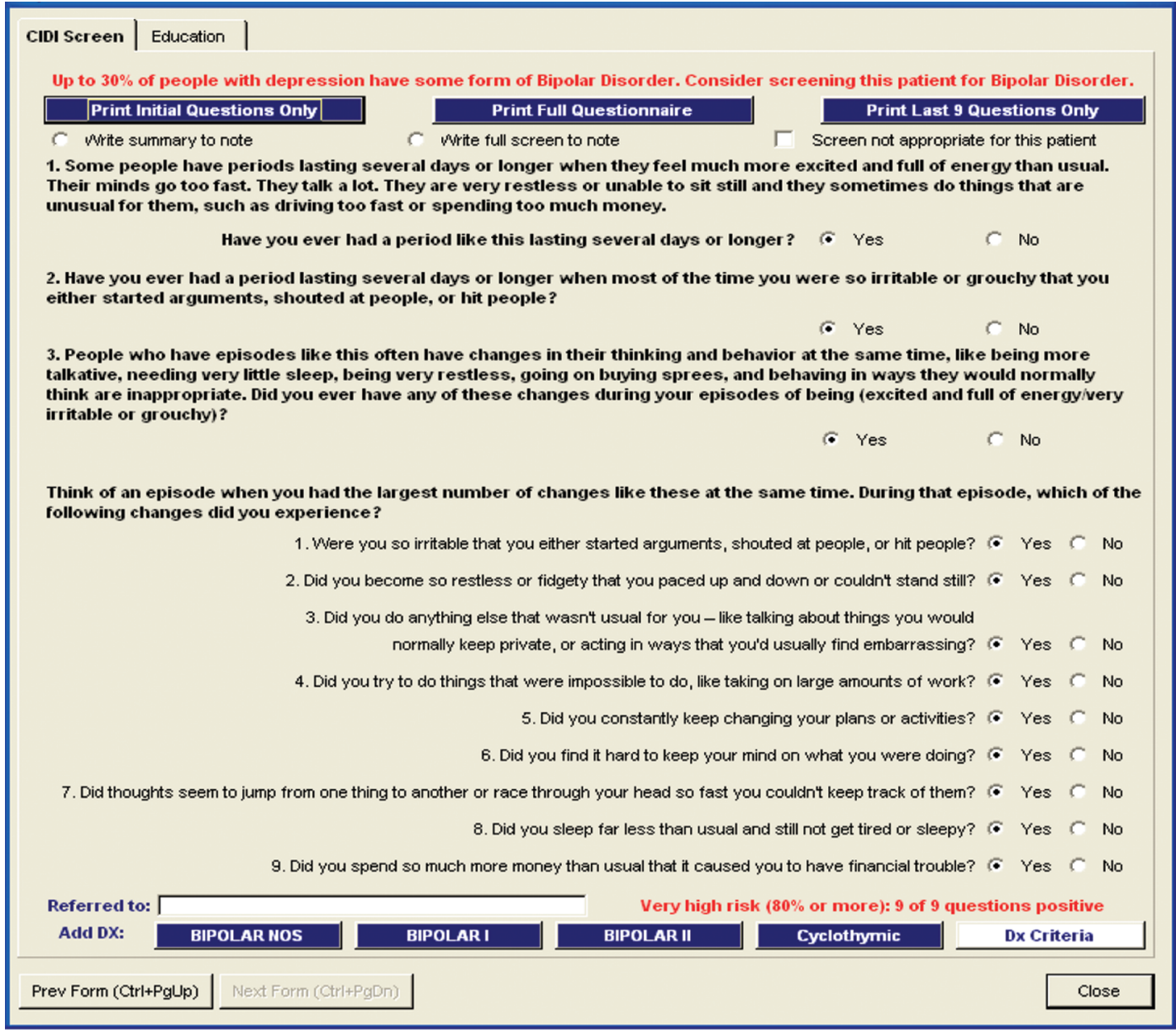

tained in the World Health Organization Composite International Diagnostic Interview (CIDI). ${ }^{25}$ This screening instrument automatically displayed during each office visit for a patient with a diagnosis of depression. The instrument was designed to be printed by the nurse or medical assistant and then self-administered by the patient, with the patient responses entered into the EHR directly on the form by the assistant for viewing by the clinician. Offices could choose a different workflow, for example, the clinician could print the form and enter responses themselves or could ask patients the questions directly; we did not track the extent to which offices used these alternative workflows.

The instrument started with 2 stem questions (see Figure 1). If the patient answered "no" to both questions, the screen was considered negative. If there was a "yes" response to either of the first 2 questions, the patient answered a third question. If the response to this third question was "no," the screen was considered negative. If the response to this third question was "yes," the patient answered 9 additional questions. Once the responses to these 9 questions were entered, the form automatically calculated the "risk" that the patient had bipolar disorder on the basis of previous validity studies ${ }^{25}$ and ranging from "very low risk" (0 to 4 questions answered "yes") to "very high risk" (all 9 questions answered "yes").

Once the risk level was displayed on the form, the clinician had the option to enter a diagnosis of bipolar disorder by clicking a button on the form. The clinician also had the option to enter free-text information about referrals to psychiatrists or other mental health specialists. Finally, the clinician had the option to obtain brief educational information about bipolar disorder directly from the form, including criteria for diagnosis and recommended 
medications and dosages for different bipolar diagnoses. They also had the option to link directly on-line to selected resources for treatment guidelines and to published articles about bipolar disorder. The manuscripts were published in a special issue of a primary care journal dedicated to bipolar disorder and were provided to the intervention clinicians before the study. ${ }^{26-30}$ Finally, the clinician had the option of printing patient education handouts regarding bipolar disorder.

Once the initial bipolar screening was completed for a patient, the EHR screening form did not automatically display at future visits. However, the form could be manually loaded if the clinician wanted to ask bipolar screening questions again or wanted to access patient or clinician educational materials. In all cases, clinicians were free to use the form or not use the form as they saw fit.

\section{Outcomes and Analysis}

The main outcomes were the rates of new diagnosis of bipolar disorder and rates of new medication prescriptions for bipolar disorder. New diagnoses were defined by an initial occurrence of a diagnosis of bipolar disorder (using ICD9 codes listed above) entered into the EHR problem list during the 6-month study period. New prescriptions were defined by a prescription for one or more medications commonly used to treat bipolar disorder that were not on the patient's medication list at the start of the study; these included carbamazepine, lamotrigine, lithium, valproate, olanzapine, quietiapine, risperidone, ziprasidone, clozapine, and apipirazole. We also examined the rate of bipolar medications specifically for patients with a new diagnosis of bipolar.

For each of these outcomes, rates were compared for intervention and comparison groups using the $\chi^{2}$ test. In addition to these main outcomes, we also calculated the percentage of patients for whom the screening tool was used as well as the results of the screen in those patients.

\section{Results}

As shown in Table 1, participating clinicians primarily worked in suburban family medicine practices and had more than 10 years of practice experience. Clinicians in the intervention group were more likely to practice in the suburbs, whereas comparison clinicians were more likely to practice
Table 1. Clinician Characteristics

\begin{tabular}{|c|c|c|}
\hline Clinician & $\begin{array}{l}\text { Intervention } \\
\quad(\mathrm{n}=75)\end{array}$ & $\begin{array}{l}\text { Control } \\
(\mathrm{n}=81)\end{array}$ \\
\hline \multicolumn{3}{|l|}{ Sex } \\
\hline Male & 52 & 58 \\
\hline Female & 48 & 42 \\
\hline \multicolumn{3}{|l|}{ Race } \\
\hline White & 87 & 90 \\
\hline Asian & 9 & 3 \\
\hline Other & 4 & 7 \\
\hline \multicolumn{3}{|l|}{ Clinician } \\
\hline Physician & 88 & 89 \\
\hline $\begin{array}{l}\text { Nurse practitioner or physician } \\
\text { assistant }\end{array}$ & 12 & 11 \\
\hline \multicolumn{3}{|l|}{ Specialty } \\
\hline Family Medicine & 76 & 88 \\
\hline Internal Medicine & 24 & 11 \\
\hline Other & 0 & 1 \\
\hline \multicolumn{3}{|l|}{ Years since residency* } \\
\hline$<2$ & 5 & 5 \\
\hline $2-5$ & 16 & 7 \\
\hline $6-10$ & 12 & 37 \\
\hline $11-30$ & 60 & 48 \\
\hline$>30$ & 7 & 3 \\
\hline \multicolumn{3}{|l|}{ Practice location* } \\
\hline Urban & 9 & 30 \\
\hline Suburban & 79 & 58 \\
\hline Rural & 12 & 12 \\
\hline
\end{tabular}

Values provided as \%.

${ }^{*} P<.01$ for difference between intervention and control groups.

in urban locations $(P<.01)$. Intervention clinicians also were more likely to be in practice more than 10 years $(P<.01)$.

The intervention clinicians had 66,575 active adult patients whereas the comparison clinicians had 71,330 active adult patients. Of these, 1018 intervention patients ( $1.53 \%$ of all adult patients) and 863 comparison patients $(1.21 \%$ of all adult patients) already had a diagnosis of bipolar disorder at the beginning of the study, so were not included in the study population. Of the active adult patients, 8355 intervention patients $(12.5 \%$ of all adult patients) and 8799 comparison patients (12.3\% of all adult patients) had a diagnosis of unipolar depression but no diagnosis of bipolar disorder at the beginning of the study and so composed the study population. Intervention group patients were more likely than comparison group patients to be aged 60 years and older 
Table 2. Primary Outcomes for Intervention and Comparison Patients

\begin{tabular}{lccrrrrr}
\hline \multirow{2}{*}{ Outcome } & \multicolumn{2}{c}{ Intervention } & & \multicolumn{2}{c}{ Comparison } & \\
\cline { 2 - 3 } & Patient Population & $\mathrm{n}(\%)$ & & Patient Population & $\mathrm{n}(\%)$ & $P$ \\
\hline New diagnoses of bipolar disorder & 8335 & $93(1.11)$ & & 8799 & $32(0.36)$ & $<.01$ \\
New bipolar medication & 7884 & $146(1.85)$ & & 8370 & $100(1.19)$ & $<.01$ \\
\hline
\end{tabular}

(32.7\% vs. $25.3 \% ; P<.0001)$ and men $(27.3 \%$ vs. $25.8 \% ; P<.05)$.

Table 2 shows the results for the main study outcomes. In the intervention group, 93 patients $(1.11 \%)$ were newly diagnosed with bipolar disorder in the 6-month study period compared with 32 patients $(0.36 \%)$ in the comparison group $(P<$ $.01)$. Regarding medications, $1.85 \%$ of the intervention patients and $1.19 \%$ of the comparison patients had a new prescription for a bipolar disorder medication entered into the EHR $(P<.01)$. For those with a new diagnosis of bipolar disorder, a higher proportion of intervention patients were prescribed medication compared with comparison patients (59.1\%, vs. $43.8 \%)$, but this difference did not achieve statistical significance $(P>.1)$.

The bipolar disorder screening tool was used in 3969 of the 8355 intervention group patients (47.5\%) during the 6-month study period. Of these, $184(2.2 \%)$ were classified as inappropriate for screening. Of the 3785 patients who completed screening, 261 (6.9\%) answered yes to either questions 1 or 2, answered yes to question 3, and completed all the CIDI screening questions. Results of screening for these 261 patients are summarized in Table 3. Ninety-eight patients ( $2.5 \%$ of all patients screened) were assessed as being at high or very high risk of bipolar disorder. As expected, these patients were more likely to be formally diagnosed with bipolar disorder, with $42 \%$ of high risk and $63 \%$ of very high risk patients diagnosed by clinicians. Clinicians also diagnosed $24 \%$ of moderaterisk and $8 \%$ of low-risk patients with bipolar disorder.

\section{Discussion}

Our intent in this study was to examine the impact of introducing an efficient EHR-based screening tool for bipolar disorder into primary care practices. The screening strategy employed in this study could be labeled "secondary screening" because it was targeted at a high-risk group that already carried a potentially inaccurate diagnosis of depression. We felt that this strategy would minimize practice burden and at the same time increase the case-finding "yield."

Our results generally supported the clinical utility of the screening tool. The tool was used by clinicians to screen nearly half of all patients with depression who did not already have a diagnosis of bipolar disorder. Although one could argue that screening rates should be even higher than that, having half of patients screened in a 6-month period is high when compared with rates of other screening behaviors that are considered "routine. $" 21,31,32$

Of those patients who were screened, $2.5 \%$ were found to be at high or very high risk for bipolar disorder according to their responses to the CIDIbased screening protocol. This proportion is lower than the rate of $3 \%$ to $24 \%$ reported in previous studies $^{7,8}$ as well as the rate of $28.4 \%$ seen in secondary screening of 269 consecutive primary care patients referred to a depression disease management program led by one of the authors (MK). Some of the differences may be because of differences in case-finding methods. Smith et $\mathrm{al}^{7}$ used a

Table 3. Results of Screening for Bipolar Disorder

\begin{tabular}{|c|c|c|}
\hline \multicolumn{2}{|l|}{ Patient Outcomes } & $\begin{array}{c}\text { Intervention Patient } \\
\text { Population }(\mathrm{n}=8355), \\
\mathrm{n}(\%)\end{array}$ \\
\hline \multicolumn{2}{|c|}{ Screened for bipolar disorder } & $3,969(47.5)$ \\
\hline \multicolumn{2}{|c|}{ Bipolar screen not appropriate } & $184(2.2)$ \\
\hline \multicolumn{2}{|c|}{$\begin{array}{l}\text { Completed CIDI screening } \\
\text { questions }\end{array}$} & $261(3.1)$ \\
\hline \multicolumn{2}{|c|}{ Accessed quick answers } & $34(0.04)$ \\
\hline CIDI Risk Level & CIDI Score & $\begin{array}{l}\text { Patients Completing } \\
\text { CIDI }(\mathrm{n}=261), \mathrm{n}(\%)\end{array}$ \\
\hline Very low risk & $0-4$ & $86(33)$ \\
\hline Low risk & 5 & $36(13.8)$ \\
\hline Moderate risk & 6 & $41(15.7)$ \\
\hline High risk & 7 or 8 & $74(28.4)$ \\
\hline Very high risk & 9 & $24(9.2)$ \\
\hline
\end{tabular}

CIDI, Composite International Diagnostic Interview. 
complex sampling strategy and administered the Hypomania Checklist, Bipolar Spectrum Diagnostic Scale, and Mini International Neuropsychiatric Interview to establish a diagnosis; on the basis of assumptions about rates of disorder in patients who did not complete screening, their case rate varied from $3.3 \%$ (most conservative) to $21.6 \%$ (least conservative). Tafalla et $\mathrm{al}^{8}$ screened primary care patients "with a current major depressive episode" with the Mood Disorder Questionnaire, did not conduct confirmatory interviews, employed a cutoff rate for "caseness" similar to that used in this study, and reported a higher rate of $24 \%$. However, their sample had a high score on the Hamilton Rating Scale for Depression at entry, suggesting that this study enrolled severely and actively depressed patients. Finally, the high rate reported in our series of 269 depressed primary care patients was obtained from a sample of more severely and chronically depressed patients who were referred to a depression disease management program. ${ }^{33}$

In contrast, this study included all patients with any current active diagnosis of depression, and therefore included the full spectrum of depression seen in primary care-from patients with minor depression to those with a single episode now in remission to those with recurrent major depressive disorder or chronic, treatment-resistant depression. This is a more representative sample of the full range of "depressed" patients seen in a typical primary care practice who would be subject to a screening protocol for bipolar disorder. Therefore, we believe that our "positive" screening rate of $2.5 \%$ is a more accurate estimate of the potential yield of this type of protocol than the higher estimates obtained by others. Although this yield seems high relative to the yield for other screening behaviors, it could be that some clinicians do not find this yield high enough to warrant spending their time on routine screening, especially for patients with more mild depression.

As expected, we found that use of the tool by clinicians resulted in a higher rate of new diagnoses of bipolar disorder; the rate of new diagnoses in the intervention group was almost triple that in the control group. The absolute rate of new diagnoses was still relatively low, however, with only $1.1 \%$ of intervention patients with depression being diagnosed with bipolar disorder. It is important to note that the formal diagnosis rate was lower than the rate of "positive" (high or very high risk) screens.
We believe this reflects the reluctance of primary care clinicians to make a definitive diagnosis without further evaluation and confirmation by a mental health specialist, especially when the results were less than conclusive. Although almost two thirds of patients with very high risk scores were diagnosed with bipolar by their primary care physician, this was true for only $42 \%$ of patients with high risk scores and $24 \%$ of patients with moderate risk scores. It is likely that primary care physicians would be more likely to defer to a specialist for making a definitive diagnosis in patients with more uncertain scores.

The rate of new bipolar medication prescriptions also was higher in the intervention group, although neither the relative difference nor the absolute difference was as great as for bipolar diagnoses. This may reflect an additional reluctance on the part of primary care physicians to initiate treatment for bipolar disorder and to instead defer treatment to a psychiatrist.

These results must be interpreted in light of study limitations. The primary limitations stem from our reliance on data that could be extracted reliably from the EHR. All mental health diagnoses were extracted from the EHR problem list and were not clinically validated, so some of the depressed patients who were identified as "high risk" for bipolar disorder might in fact have an established bipolar diagnosis not known to the primary care clinician. This is particularly true when patients were referred to mental health specialists who then made the diagnosis of bipolar disorder; though clinicians could indicate a referral on the form, this was done in text comments that were not tracked. Also, we do not know for sure that "bipolar medications" were in fact used for bipolar disorder because some of these medications can be used for other purposes. Because these medication prescriptions were extracted from the record and could not be validated against pharmacy records, we were, in effect, assessing clinicians' initial treatment decisions. We could not explore the context of those diagnostic or treatment decisions, nor could we explore the nuanced management plans that clinicians may have used in uncertain clinical situations. In some cases, provisional diagnoses or use of sample medications would have been included in a text visit note but not in the data fields we could access. However, our primary aim was to assess the impact of the CDS tool on diagnosis and treatment deci- 
sions, and we believe that diagnoses and medications recorded in the EHR provide a reasonable and valid way to assess those decisions. Also, limitations in the validity of diagnoses or medications are unlikely to differ between the intervention and comparison physicians, and differences between the 2 groups are unlikely to be effected by the limitations of EHR data. We also acknowledge that the study included only clinicians who use one particular EHR, who participate in the CHURN PBRN, and who agreed to participate in this particular PBRN study. One study found that patients in the MQIC database used by CHURN to be similar to the general US population in terms of disease prevalence, ${ }^{34}$ but studies have not examined differences between the CHURN or MQIC populations and other outpatient populations in terms of quality of care. It is not clear whether these results can be generalized to apply to clinicians practicing in different environments.

\section{Conclusions}

There is increasing recognition that bipolar disorder is both common and underrecognized in primary care settings. ${ }^{3,7}$ Efforts to improve quality of care could focus on improving rates of detection, increasing rates of treatment by primary care clinicians, or providing enhanced specialty-level resources for treatment. Although all 3 will be important, improved detection is a necessary first step. To our knowledge, this is the first study to explore the usefulness of EHR-prompted "secondary screening" for bipolar disorder among high-risk primary care patients. Our core findings of widespread use of the CDS tool and a higher rate of diagnosis and medication prescription suggest that EHR-based CDS can be useful in improving the detection of bipolar disorder in patients with depression. This selective screening approach, coupled with expanded versions of the stepped collaborative-care interventions that have proven so successful for primary care depression treatment, ${ }^{33,35-37}$ could significantly improve care for bipolar disorder in the primary care setting.

The authors would like to thank the physicians and practices of the Centricity Health care User Research Network (CHURN) that participated in this study. We thank the project manager, Ms. Katherine Rosch Hegedus, and Ms. Cheryl Mongillo for her assistance with manuscript preparation.

\section{References}

1. Loganathan M, Lohano K, Roberts RJ, Gao Y, ElMallakh RS. When to suspect bipolar disorder. J Fam Pract 2010;59(12):682-8.

2. Ghaemi SN, Boiman EE, Goodwin FK. Diagnosing bipolar disorder and the effect of antidepressants: a naturalistic study. J Clin Psych 2000;61(10):804-8; quiz 809.

3. Smith DJ, Ghaemi N. Is underdiagnosis the main pitfall when diagnosing bipolar disorder? Yes. BMJ 2010;340:c854.

4. El-Mallakh RS, Karippot A. Chronic depression in bipolar disorder. Am J Psychiatry 2006;163(8):133741; quiz 1478.

5. Klinkman MS, Okkes I. Mental health problems in primary care a research agenda. J Fam Pract 1998; 47(5):379-84.

6. Ford DE. Managing patients with depression: is primary care up to the challenge? J Gen Intern Med 2000;15(5):344-5.

7. Smith DJ, Griffiths E, Kelly M, Hood K, Craddock $\mathrm{N}$, Simpson SA. Unrecognised bipolar disorder in primary care patients with depression. Br J Psychiatry 2011;199:49-56.

8. Tafalla M, Sanchez-Moreno J, Diez T, Vieta E. Screening for bipolar disorder in a Spanish sample of outpatients with current major depressive episode. J Affect Disord 2009;114(1-3):299-304.

9. Klinkman MS. Competing demands in psychosocial care. A model for the identification and treatment of depressive disorders in primary care. Gen Hosp Psychiatry 1997;19(2):98-111.

10. Nutting PA, Rost K, Smith J, Werner JJ, Elliot C. Competing demands from physical problems: effect on initiating and completing depression care over 6 months. Arch Fam Med 2000;9(10):1059-64.

11. Chambers CV, Balaban DJ, Carlson BL, Ungemack JA, Grasberger DM. Microcomputer-generated reminders. Improving the compliance of primary care physicians with mammography screening guidelines. J Fam Pract 1989;29:273-80.

12. Chambers CV, Balaban DJ, Carlson BL, Grasberger DM. The effect of microcomputer-generated reminders on influenza vaccination rates in a university-based family practice center. J Am Board Fam Pract 1991;4(1):19-26.

13. McDonald CJ, Hui SL, Smith DM, et al. Reminders to physicians from an introspective computer medical record. A two-year randomized trial. Ann Intern Med 1984;100:130-8.

14. Ornstein SM, Garr DR, Jenkins RG, Rust PF, Arnon A. Computer-generated physician and patient reminders. Tools to improve population adherence to selected preventive services. J Fam Pract 1991;32(1): 82-90.

15. Gill JM, Dansky B. Use of an electronic medical record to facilitate screening for depression in pri- 
mary care. Prim Care Companion J Clin Psychiatry. 2003;5(3):125-8.

16. Klein EW, Hunt JS, LeBlanc BH. Depression screening interfaced with an electronic health record: a feasibility study in a primary care clinic using optical mark reader technology. Prim Care Companion J Clin Psychiatry 2006;8(6):324-8.

17. Rollman BL, Hanusa BH, Lowe HJ, Gilbert T, Kapoor WN, Schulberg HC. A randomized trial using computerized decision support to improve treatment of major depression in primary care. J Gen Intern Med 2002;17(7):493-503.

18. Gill J, Chen YX. Quality of lipid management in outpatient care: a national study using electronic health records. Am J Med Qual 2008;23(5):375-81.

19. Gill JM, Chen YX, Lieberman MI. Management of depression in ambulatory care for patients with medical co-morbidities: a study from a national electronic health record (EHR) network. Int J Psychiatry Med 2008;38(2):203-15.

20. Gill JM, Fleischut P, Haas S, Pellini B, Crawford A, Nash DB. Use of antibiotics for adult upper respiratory infections in outpatient settings: a national ambulatory network study. Fam Med 2006;38(5): 349-54.

21. Gill JM, Foy AJ, Ling Y. Quality of outpatient care for diabetes mellitus in a national electronic health record network. Am J Med Qual. 2006;21(1):13-7.

22. Gill JM, Klinkman MS, Chen YX. Antidepressant medication use for primary care patients with and without medical comorbidities: A National Electronic Health Record (EHR) Network study. J Am Board Fam Med 2010;23(4):499-508.

23. Gill JM, Chen YX, Glutting JJ, Diamond JJ, Lieberman MI. Impact of decision support in electronic medical records on lipid management in primary care. Popul Health Manag 2009;12(15):221-6.

24. Gill JM, Landon BE, Antonelli RC, Rich EC. Generating the knowledge needed to make the patientcentered medical home a reality: a collaborative project of the primary care specialties. Ann Fam Med 2010;8(1):88-9.

25. Kessler RC, Akiskal HS, Angst J, et al. Validity of the assessment of bipolar spectrum disorders in the WHO CIDI 3.0. J Affect Disord 2006;96(3): 259-69.

26. Defining the challenge: recognizing and treating bipolar disorder wherever patients present. J Fam Pract 2007;56(11 Suppl 1):S3-4.

27. Recognizing and understanding bipolar disorder. Part 2. J Fam Pract 2007;56(11 Suppl 1):S5-10.

28. Clinical management of bipolar disorder: role of the primary care provider. J Fam Pract 2007;56(11 Suppl 1):S11-8.

29. Treatment by phase: pharmacologic management of bipolar disorder. J Fam Pract 2007;56(11 Suppl 1): S19-27.

30. Recognizing bipolar disorder on initial presentation: a case study with decision points. J Fam Pract 2007; 56(11 Suppl 1):S28-32.

31. Gill J. Physician performance of preventive care for women. Del Med J. 1996;68(7):349-55.

32. Gill JM, Chen Y. Quality of lipid management in outpatient care: a national study using electronic health records. Am J Med Qual. 2008;23(5):375-81.

33. Klinkman MS, Bauroth S, Fedewa S, et al. Longterm clinical outcomes of care management for chronically depressed primary care patients: a report from the Depression in Primary Care Project. Ann Fam Med 2010;8(5):387-96.

34. Oderda G, Brixner D, Lieberman M. Comparison of EMR data to US national data. Paper presented at: International Society for Pharmacoeconomics and Outcomes Research (ISPOR), 10th Annual European Congress, 2007; Dublin, Ireland.

35. Katon W, Russo J, Von Korff M, et al. Long-term effects of a collaborative care intervention in persistently depressed primary care patients. J Gen Intern Med. Oct 17(10):741-748, 2002.

36. Unutzer J, Rubenstein L, Katon WJ, et al. Two-year effects of quality improvement programs on medication management for depression. Arch Gen Psychiatry. Oct 58(10):935-942, 2001.

37. Katon W, Unützer J, Wells K, Jones L. Collaborative depression care: history, evolution and ways to enhance dissemination and sustainability. General Hospital Psychiatry. 2010/10// 32(5):456-464, 2010. 